\title{
Studio di prevalenza batterica e fungina su espettorati provenienti da pazienti con Fibrosi Cistica
}

\author{
Marina Brugia, Patrizio Frontini \\ Laboratorio Analisi Ospedale Pediatrico ad alta specializzazione G. Salesi \\ Azienda Ospedaliero - Universitaria Ospedali Riuniti Umberto I - G.M. Lancisi- G. Salesi Ancona
}

Key words: Cystic fibrosis, control infection, antibiotic-resistance

Study of bacterial ad fungal prevalence in sputum of patients with cystic fibrosis

\section{SUMMARY}

Over the past 20 years there has been a grater interest in infection control in cystic fibrosis (CF). The epidemiology of pathogens in CF patients has become more complex; many common and complex pathogens species may be pathogens in patients with CF.

In addition the number of different bacterial species isolated from respiratory specimens from this patient population are also numerous. The main objective when treating people who have CF is to prevent, eradicate or control all types of respiratory infection, particularly endobronchial and pulmonary infection.

We examined 475 sputum of 69 patients with FC during 2003. Pseudomonas aeruginosa was prevalent, occurs in $85 \%$ of CF patients studied. The mucoid phenotype was isolated in $44,4 \%$ of patients. The prevalence of S. maltophilia, A. xylosoxidans, Burkholderia cepacia and Aspergillus spp. in our patients is $1,8 \%, 2,1 \%, 2,9 \%$ and $26,1 \%$ respectively.

$\mathrm{CF}$ isolates are tested for antibiotic susceptibility for control of antimicrobial resistance and the emergence of multidrug-resistant organism. The choice of antibiotic depends on in vitro sensitivity pattern. The regular microbiological monitoring can contribute to extend life for many CF patients but also contribute to improving the quality of life for this population.

\section{INTRODUZIONE}

La Fibrosi Cistica (FC) è una delle malattie ereditarie più frequenti nella popolazione caucasica. I soggetti che possono trasmettere la malattia, portatori asintomatici, sono circa il 5\% della popolazione, poiché la malattia si manifesta solo allo stato di omozigote, si calcola che nasca affetto un bambino su 2500-3000 nati.

È una malattia cronica, evolutiva causata dall'alterazione di una proteina CFTR che porta ad un'anomalia delle secrezioni mucose ed enzimatiche che risultano più dense e vischiose con progressiva compromissione dell'apparato respiratorio e/o digestivo.

Le aspettative di vita per questi pazienti sono in continuo aumento: nel 2001 l'età media di sopravvivenza in America è stata calcolata di 33,4 anni e il $37 \%$ dei pazienti aveva un'età $>$ di 18 anni (Cystic Fibrosis Foundation 2002).

Questo ha portato, negli ultimi anni, ad un maggior interesse per il controllo, l'epidemiologia delle infezioni in questi soggetti e ad un maggior coinvolgimento del laboratorio microbiologico nella diagnosi.

I patogeni principali continuano ad essere: Staphylococcus aureus, Haemophilus influenzae, Pseudomonas aeruginosa, ma iniziano ad avere un ruolo importante dei patogeni emergenti quali Burkholderia cepacia complex, Stenotrophomonas maltophilia, Alcaligenes xylosoxidans, Aspergillus spp. (Saiman 2004).

\section{SCOPO DEL LAVORO}

Scopo del presente lavoro è di valutare l'epidemiologia e la prevalenza dei vari microrganismi isolati da espettorati di pazienti con Fibrosi cistica nella Regione Marche e di sorvegliare la resistenza di tali ceppi fornendo ai clinici dati utili per un applicazione terapeutica più appropriata ed efficace.

Il controllo longitudinale dell'esame microbiologico nel singolo paziente può consentire inoltre di valutare la comparsa nell'escreato di nuovi batteri e funghi e verificare l'aumento della carica microbica così da fornire utili indicazioni su un eventuale loro ruolo di patogenicità.

La valutazione infine dei risultati ottenuti ci fornisce importanti informazioni di tipo epidemiologico che consentono il confronto con altri Centri.

\section{MATERIALI E METODI}

Nell'anno 2003 sono stati esaminati 475 escreati provenienti da 69 pazienti seguiti presso il centro regionale di Fibrosi cistica della nostra Azienda.

Di questi $33(47,8 \%)$ hanno un'età $\leq$ a 18 anni e $36(52,2 \%)$ un'età $>18$ anni.

La media dei campioni inviati è stata di 9 per ciascun paziente. 
La semina di tutti i campioni è stata fatta contemporaneamente su Agar cioccolato, Columbia CNA, MSA, Mc Conkey, Albicans ID e BCSA (Oxoid). Il materiale è stato diluito $1: 1(\mathrm{v} / \mathrm{v})$ con Ditiotreitolo (tecnica semiquantitativa), incubato a $37^{\circ} \mathrm{C}$ per $30^{\prime}$ e seminato secondo la tecnica delle tre zone.

L'incubazione è stata fatta in aerobiosi a una temperatura di $35-37^{\circ} \mathrm{C}$ per 48 ore, fino ad un massimo di 5 giorni per i microrganismi a crescita più lenta (Burkholderia cepacia).

L'identificazione dei ceppi è stata eseguita mediante Vitek e sistema API (bioMèrieux). Per il test di sensibilità agli antibiotici la tecnica utilizzata è stata sia quella in agar secondo KirbyBauer ponendo i dischi con antibiotico (Oxoid) su piastra Muller-Hinton con sospensione preparata direttamente dalle colonie cresciute in coltura, sia quella automatizzata (Vitek).

Non sono stati ricercati micobatteri atipici e batteri anaerobi.

\section{RISULTATI}

Dei 69 pazienti sono stati esaminati 475 escreati di cui 83 negativi $(17,4 \%)$, gli isolati riscontrati e le rispettive percentuali sono riportate nella tabella 1 .

Tabella I. Frequenza isolamento microrganismi anno 2003

\begin{tabular}{lcc}
\hline IDENTIFICAZIONE ORGANISMO & $\%$ & N. ISOLATI \\
\hline Acinetobacter Iwoffii & 1,9 & 12 \\
\hline Alcaligenes specie & 1,0 & 6 \\
\hline Alcaligenes xylosoxidans & $2, \mathrm{I}$ & 13 \\
\hline Aspergillus fumigatus & 7,1 & 44 \\
\hline Bacilli Gram negativi non fermentanti & 1,8 & $1 \mathrm{I}$ \\
\hline Branhamella catarrhalis & 0,2 & $\mathrm{I}$ \\
\hline Burkolderia cepacia & 1,3 & 8 \\
\hline Candida specie & 3,2 & 20 \\
\hline Citrobacter freundii & 0,2 & 1 \\
\hline Enterobacter cloacae & 0,2 & 1 \\
\hline Escherichia coli & $1, \mathrm{I}$ & 7 \\
\hline Haemophylus influenzae & 3,1 & 19 \\
\hline Klebsiella & 0,6 & 4 \\
\hline Pseudomonas aeruginosa & $4 \mathrm{I}, 6$ & 259 \\
\hline Serratia marcescens & 0,5 & 3 \\
\hline Staphylococcus aureus & 32,0 & 199 \\
\hline Stenotrophomonas maltophilia & 1,8 & $1 \mathrm{I}$ \\
\hline Streptococcus pyogenes & 0,3 & 2 \\
\hline Streptococcus pneumoniae & 0,2 & 1 \\
\hline TOTALE & 100,0 & 622 \\
\hline
\end{tabular}

Sono stati isolati 259 ceppi di Pseudomonas aeruginosa di cui $115(44,4 \%)$ di tipo mucoide e 95 $(36,6 \%)$ di tipo rugoso. La sensibilità agli antibiotici di questi ceppi è mostrata in figura I. La sensibilità dei ceppi di Staphylococcus aureus isolati è mostrata nella figura II.
Tra i microrganismi emergenti sono stati isolati 13 ceppi di Alcaligenes xylosoxidans (2,1\%), 11 di Stenotrophomonas maltophilia $(1,8 \%)$ e 8 di Burkholderia cepacia complex (1,3\%). La sensibilità in vitro di questi ceppi sono rappresentati nella figura III, IV, V.

\section{DISCUSSIONE E CONCLUSIONI}

La quasi totalità dei pazienti studiati presentano più batteri nello stesso campione e data la cronicità delle infezioni, la comparsa intermittente di alcuni batteri.

Come riportato in tabella 1 Pseudomonas aeruginosa è stato isolato con maggiore frequenza, 1 ' $85 \%$ dei pazienti (58) sono infettati da questo patogeno.

$P$. aeruginosa ha una straordinaria capacità di mettere in opera sistemi di regolazione per modulare le proprie capacità metaboliche in relazione alle caratteristiche ambientali.

È pertanto particolarmente difficile da trattare oltre che per la le sue capacità di resistenza naturale, per la capacità di eludere o eliminare gli antibiotici.

Alcuni lavori hanno dimostrato che pazienti con FC infettati cronicamente con $P$. aeruginosa hanno una sopravvivenza inferiore di circa 10 anni.

Il monitoraggio microbiologico regolare di questi soggetti è importante perché può aiutarci ad identificare l'insorgenza della cronicità e la presenza di infezioni respiratorie che se trattate precocemente possono essere eradicate (Doring 2000, Rotjen 2001).

Nella nostra casistica come mostra la figura I si evidenzia una maggiore percentuale di resistenza alla gentamicina, netilmicina, amikacina e tobramicina.

Il fenotipo mucoide, spesso associato a prognosi sfavorevole, risulta essere isolato nel 43,5\% dei pazienti (30). In questi isolati non si evidenziano, in vitro, delle farmacoresistenze in vitro particolarmente elevate, pertanto la non responsività alla terapia antibiotica e la prognosi sfavorevole di tali pazienti è probabilmente da ricercare in meccanismi alternativi tra i quali l'esagerata risposta immunitaria (proteasi alcalina, elastasi) inefficace ad eliminare $i$ batteri ma in grado di compromettere la funzionalità delle vie aeree.

Staphylococcus aureus è stato il primo patogeno polmonare riconosciuto nei pazienti con FC, circa il $50 \%$ dei pazienti sono infettati e l'infezione compare abbastanza precocemente.

Il trattamento prolungato con antibiotici non ha dimostrato nessun significativo vantaggio anzi la profilassi anti-stafilococcica può aumentare l'incidenza di $P$. aeruginosa. I ceppi meticillino-resi- 
stenti sono resistenti a tutti i $\beta$-lattamici e spesso ad aminoglicosidi e macrolidi quindi il controllo di questi ceppi è molto importante per la scelta della terapia mirata.

Nella nostra casistica 44 pazienti $(63,7 \%)$ sono risultati infettati e 12 ceppi $(6 \%)$ isolati da 2 pazienti presentavano meticillino-resistenza (figura II).

B. cepacia complex, a cui appartengono 9 specie diverse, in realtà è un patogeno studiato già dagli inizi del 1980.

È un batterio multiresistente che porta ad un rapido peggioramento della funzionalità polmonare con esiti spesso fatali per un'alta percentuale dei pazienti (Tabian 1987).

La sopravvivenza media dei pazienti infettati è notevolmente ridotta anche nei casi d'infezione cronica.

La prevenzione di quest'infezione è possibile solo con interventi coordinati in più fronti: nell'educazione dei pazienti e dei familiari, nel controllo di tutte le prassi in ambiente ospedaliero, nella decontaminazione e nel controllo dei contatti.

Nella nostra casistica in cui solo 2 pazienti $(2,9 \%)$ sono stati infettati con B. stabilis e VI B. cepacia complex, il controllo di questo microrganismo serve come prototipo per la sorveglianza delle infezioni nei pazienti con FC.

S. maltophilia e A. xylosoxidans sono i patogeni che stanno assumendo sempre più importanza nei pazienti con FC, soprattutto adulti.

Nei nostri pazienti la prevalenza è rispettivamente del 2,9\% (2 pazienti) e 3,0\% (3 pazienti) leggermente inferiore alla prevalenza americana.

La patogenicità di questi germi nei pazienti con FC non è stata ancora stabilita, anche se sono stati segnalati complicanze polmonari in associazione con questi microrganismi.

È da rilevare come si può vedere nella figura III, IV una resistenza molto elevata nei ceppi isolati con difficoltà di trattamento antibiotico.

La prevalenza d'Aspergillus spp. è nella nostra casistica del 26,1\% (18 pazienti) significativamente più elevata di quella americana $(10,6 \%)$ segnalata nel 2001.

Nessun paziente manifestava la sindrome broncopolmonare, pertanto si può ipotizzare che una percentuale così elevata sia dovuta alla profilassi antibiotica per aerosol che sembra essere il fattore di rischio maggiore per la colonizzazione fungina.

Dalla nostra esperienza ci appare molto importante il ruolo del laboratorio nel monitoraggio microbiologico continuo dei pazienti con fibrosi cistica sia in termini di scelte terapeutiche che di qualità di vita.

$\mathrm{Ci}$ appare altresì importante determinare le preva- lenze dei microrganismi per poter adottare modelli organizzativi basati sulla segregazione dei pazienti colonizzati da $P$. aeruginosa o $B$. cepacia o da ceppi multiresistenti da quelli non colonizzati per evitare così il contatto con l'agente infettante e prevenire la colonizzazione cronica. La prevalenza dei patogeni respiratori infine può aiutare nella scelta di misure specifiche di prevenzione ambientale e di segregazione.

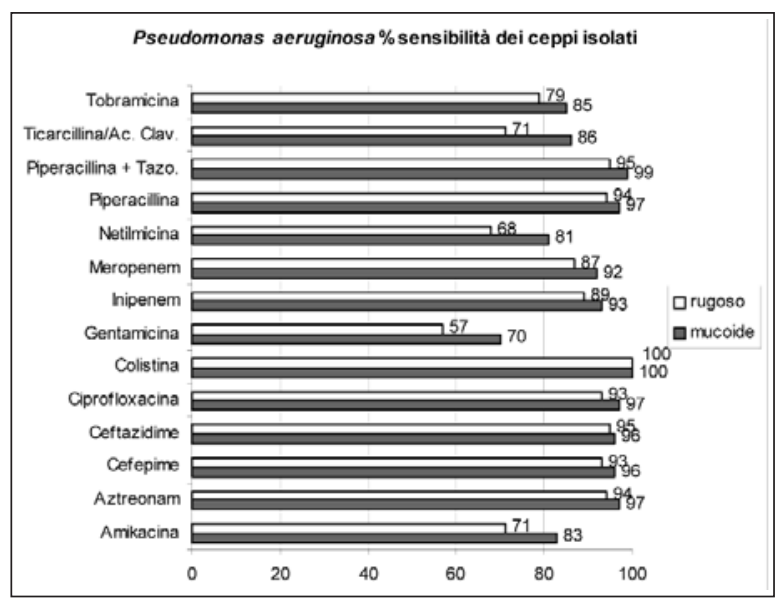

Figura I

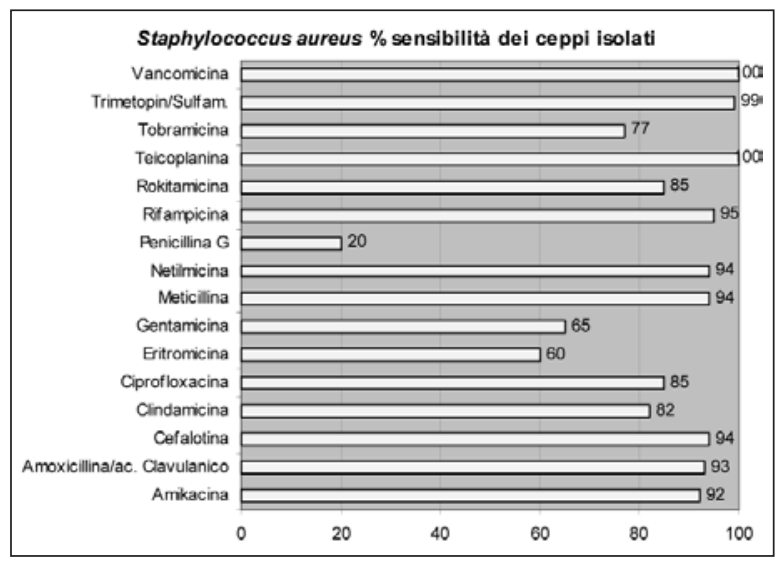

Figura II

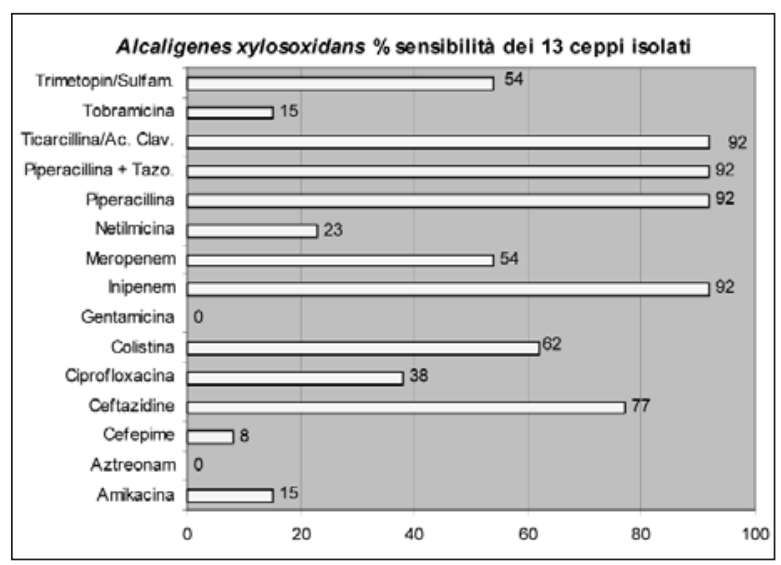

Figura III 


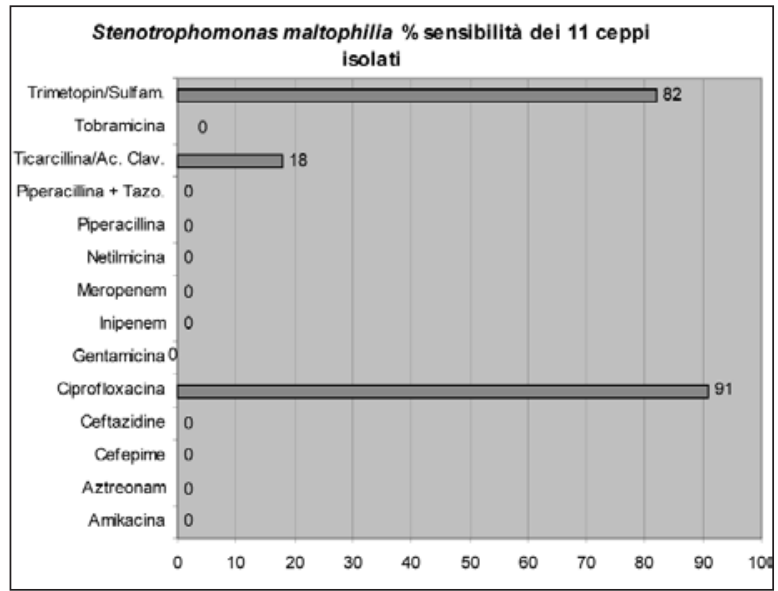

Figura IV

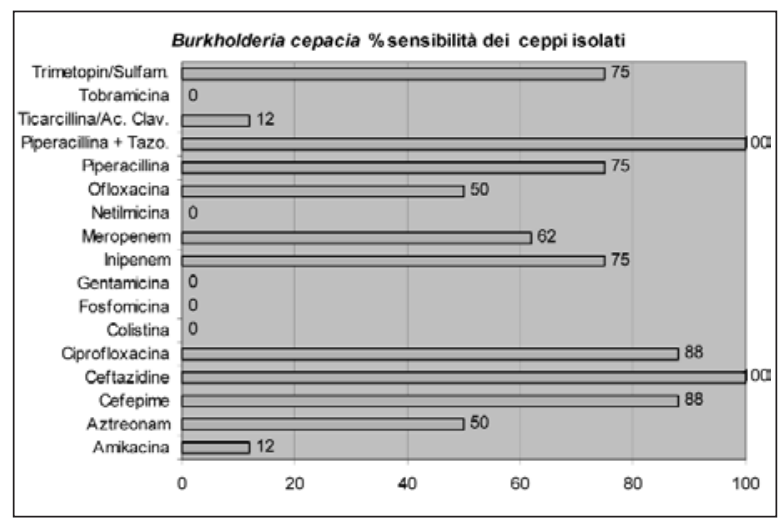

Figura V

\section{BIBLIOGRAFIA}

1. Aron SD, Ferris W, Ramotar K, et al. Single and combination antibiotic susceptibilities of planktonic, adherent, and biofilm-grown Pseudomonas aeruginosa isolates cultured from sputa of adults with cystic fibrosis. J Clin Microbiol 2002; 40: 4172-9.

2. Aris RM, Routh JC, LiPuma JJ, Heath DG, Gilligan PH. Lung transplantation for cystic fibrosis patients with Burkholderia cepacia complex. Survival linked to genomovar type. Am J Respir Crit Care Med 2001; 164: 2102-6.

3. Cystic Fibrosis Foundation. Patient registry 2001 annual report. Cystic Fibrosis Foundation, Washington, DC 2002.

4. Doring G, Conway SP, Heijermon HGM, et al. Antibiotic therapy against Pseudomonas aeruginosa in cystic fibrosis: a European consensus. Eur Respir J 2000; 16: 749-67.

5. Emerson J, Rosenfeld M, Mc Namarra S, et al. Pseudomonas aeruginosa and other predictor of mortality and morbidity in young children with cystic fibrosis. Pediatr Pulmonol 2002; 34: 91-100.

6. Lyczak JB, Cannon CL, Pier GB. Lung infections associated with cystic fibrosis. Clin Microbiol Rev 2002; 15: 194-222.

7. Lyczak JB, Cannon CL, Pier GB. Lung infections associated with cystic fibrosis. Clin Microbiol Rev 2002; 15: 194-222.

8. Miller BM, Gilligan PH. Laboratory aspects of management of chronic pulmonary infections in patients with Cystic Fibrosis. J Clin Microbiol Minireview 2001; 41: 4009 -15.

9. Ratjen F, Doring G, Nikolaizik WH. Effect of inhalated tobramycin on early Pseudomonas aeruginosa colonisation in patients with cystic fibrosis. Lancet 2001; 358: 983-4.

10. Saiman L, Burns JL, Larone D, Chen Y, Garber E, Whittier S. Evaluation of MicroScan Autoscan for identification of Pseudomonas aeruginosa isolates from cystic fibrosis patients. J Clin Microbiol 2003; 41: 492-4.

11. Saiman L, Chen Y, Tabibi S, San Gabriel P, et al. Identification and antimicrobial susceptibility of Alcaligenes xylosoxidans isolated from patients with cystic fibrosis. J Clin Microbiol 2001; 39: 3942-5.

12. Saiman L, Siegel J. Infection control in cystic fibrosis. Clin Microbiol Reviews 2004; 17: 57-71.

13. Tabian OC, Martone WC, Doersshuk CF, Stern C, et al. Colonization of the respiratory tract with Pseudomonas aeruginosa in cystic fibrosis. Risk factors and outcomes. Chest 1987; 91: 527-32.
Marina Brugia

Laboratorio Analisi Ospedale G. Salesi

Via Corridoni 11 - 60123 Ancona

Tel. 0715962294; Fax 0715962299

E-mail: m.brugia@libero.it 